\title{
BRANCHING RANDOM WALKS ON BINARY SEARCH TREES: CONVERGENCE OF THE OCCUPATION MEASURE
}

\author{
ERIC FEKETE ${ }^{1}$
}

\begin{abstract}
We consider branching random walks with binary search trees as underlying trees. We show that the occupation measure of the branching random walk, up to some scaling factors, converges weakly to a deterministic measure. The limit depends on the stable law whose domain of attraction contains the law of the increments. The existence of such stable law is our fundamental hypothesis. As a consequence, using a one-to-one correspondence between binary trees and plane trees, we give a description of the asymptotics of the profile of recursive trees. The main result is also applied to the study of the size of the fragments of some homogeneous fragmentations.
\end{abstract}

Mathematics Subject Classification. 60F05, 60G50, 68W40, 60J80, $05 \mathrm{C} 05$.

Received February 9, 2007. Revised November 13, 2007 and October 16, 2008.

\section{INTRODUCTION}

The purpose of this paper is to study the asymptotics of the occupation measure of branching random walks (BRWs) when the underlying tree is a binary search tree. This work is motivated by the large number of results on the occupation measure of BRWs on conditioned Galton-Watson trees which limit is ISE (Integrated Super-Brownian Excursion), and also by some applications in term of fragmentation processes and in term of the profile of recursive trees discussed later.

Informally, a branching walk is a random object owning two levels of randomness. First an underlying tree is picked up under a certain law and then, on each of its edges, a random variable is attached and is used as the increment of a random walk. Hence, a BRW is a family of dependent random walks indexed by the nodes of the underlying tree (it is formally defined below). Many results on BRWs can be found in the literature.

During the last thirty years, Biggins obtained many different results when the underlying tree is a supercritical Galton-Watson tree. For instance, in [3], he studied the convergence of a martingale associated to the BRW.

More recently, Aldous [1] introduced a model of BRW on trees of size $n$ : the underlying tree $\mathcal{T}$ is the family tree of a Galton-Watson process conditioned to have $n$ nodes (with offspring distribution having mean 1 and variance $\sigma^{2}$ ) and the increments are independent, centered, with variance $\theta^{2}$ and having moments of order $4+\epsilon$. With each node $u$ of $\mathcal{T}$, he associates the variable $Y_{u}$ which is the sum of the increments associated with the ancestors of $u$. Aldous stated that the normalized occupation measure converges to a random measure called

Keywords and phrases. Random binary search tree, branching random walk, occupation measure, fragmentation, recursive tree.

1 UVSQ, Département de Mathématiques, 45 av. des États-Unis, 78035 Versailles Cedex, France; fekete@math.uvsq.fr 
ISE, that is

$$
\frac{1}{n+1} \sum_{u \in \mathcal{T}} \delta_{\frac{\sigma^{1 / 2} Y_{u}}{\theta n^{1 / 4}}} \underset{n \rightarrow \infty}{\stackrel{\mathcal{D}}{\longrightarrow}} \mu_{I S E}
$$

where $\delta$ stands for the Dirac measure, in the space of probability measures. The measure $\mu_{I S E}$ owns a representation in term of Gaussian process and appears as the natural limit of many different random phenomena, see e.g. $[1,7,12,20,21]$ and references therein. In particular, Chassaing and Schaeffer [7] have shown that it is closely related to the limit of the profile of uniform quadrangulations.

By contrast with $\mu_{I S E}$, which is a random measure, we will see that things are very different on a binary search tree, as the limiting occupation measure is deterministic and equal to the stable distribution corresponding to the spatial displacements (except in the non-centered Gaussian case where variance is different).

The paper is organized as follows. In the continuation of Section 1 we define our model of branching random walk. Then we give our main results on the convergence of the occupation measure in Section 2, according to whether the increments are identically distributed or not. The proof of Theorem 2.1 is relegated to Section 4. Section 3 provides two applications of Corollary 2.1 in term of homogeneous fragmentations and in term of the profile of recursive trees.

\subsection{Binary search trees (BSTs) and finite branching random walks}

Let us introduce our model of branching random walk. For this, we begin with a formal description of trees. Let

$$
\mathbb{U}:=\{\varnothing\} \cup \bigcup_{n \in\{1,2, \cdots\}} \mathbb{N}^{n}
$$

be the set of the finite words on the alphabet $\mathbb{N}:=\{0,1,2, \cdots\}$ (with $\varnothing$ as the empty word). We write $u v$ the word formed by the concatenation of $u$ and $v$. A plane tree $\tau$ is a finite subset of $\mathbb{U}$ such that

$$
\left\{\begin{array}{l}
\varnothing \in \mathcal{T}, \\
\forall(u, v) \in \mathbb{U}^{2}, \quad \text { if } u v \in \mathcal{T} \text { then } u \in \mathcal{T}, \\
\forall u \in \mathbb{U}, \forall d \in \mathbb{N}^{*}:=\{1,2, \cdots\}, \quad \text { if } u d \in \mathcal{T} \text { then } u(d-1) \in \mathcal{T} .
\end{array}\right.
$$

The elements of $\mathcal{T}$ are called nodes, and $\varnothing$ is called the root. The number of letters in $u$, denoted by $|u|$, is called the depth of $u$ (with $|\varnothing|=0)$. We say that $v$ is an ancestor of $u$ when $v$ is a prefix of $u(\exists w \in \mathbb{U} ; v w=u)$, we denote it by $v \ll u$. We write $\mathcal{P}$ the set of plane trees. Remark that the above definition of a plane tree fits with the usual one: a plane tree is a finite rooted unlabeled loop free connected graph in which the set of children of every vertex is endowed with a total order.

In the present paper we are concerned with complete binary trees. Let $\mathbb{U}^{b}:=\{\varnothing\} \cup \bigcup_{n \geq 1}\{0,1\}^{n}$, be the subset of $\mathbb{U}$ of the finite words on the alphabet $\{0,1\}$. A complete binary tree $\mathcal{T}$ is a plane tree such that every node has zero or two children (in other words $\mathcal{T}$ is a finite subset of $\mathbb{U}^{b}$ satisfying (1.1) and such that $u 1 \in \mathcal{T} \Leftrightarrow u 0 \in \mathcal{T}$ ). We write $\mathcal{B}$ for the set of complete binary trees. A tree $\mathcal{T}$ can be described by giving the set $\partial \mathcal{T}$ of its leaves (the nodes with no descendants also called external nodes). The nodes of $\mathcal{T} \backslash \partial \mathcal{T}$ are called internal nodes.

As said above a branching random walk is a random object having two levels of randomness: the underlying tree and the increments. We first introduce our model of random underlying tree, the binary search tree (BST).

\subsection{The underlying tree model}

Random BSTs are fundamental data structures associated with quicksort, one of the most used sorting algorithms. We present a recursive construction of BSTs, or we should say labeled BSTs: $\left(U_{i}\right)_{i \geq 1}$ is a sequence of independent random variables uniformly distributed on $[0,1]$. With the $n$ first terms $\left(U_{i}\right)_{i=1 \cdots n}$ we associate a labeled BST of size $n$, that is a complete binary tree in which each of the $n$ internal nodes contains one of the values $\left(U_{i}\right)_{i=1 \cdots n}$ : the root contains $U_{1}$, the left subtree is the labeled BST associated with the sequence of the $U_{i}$ 's smaller than $U_{1}$ and the right subtree is the labeled BST associated with the sequence of the $U_{i}$ 's larger 

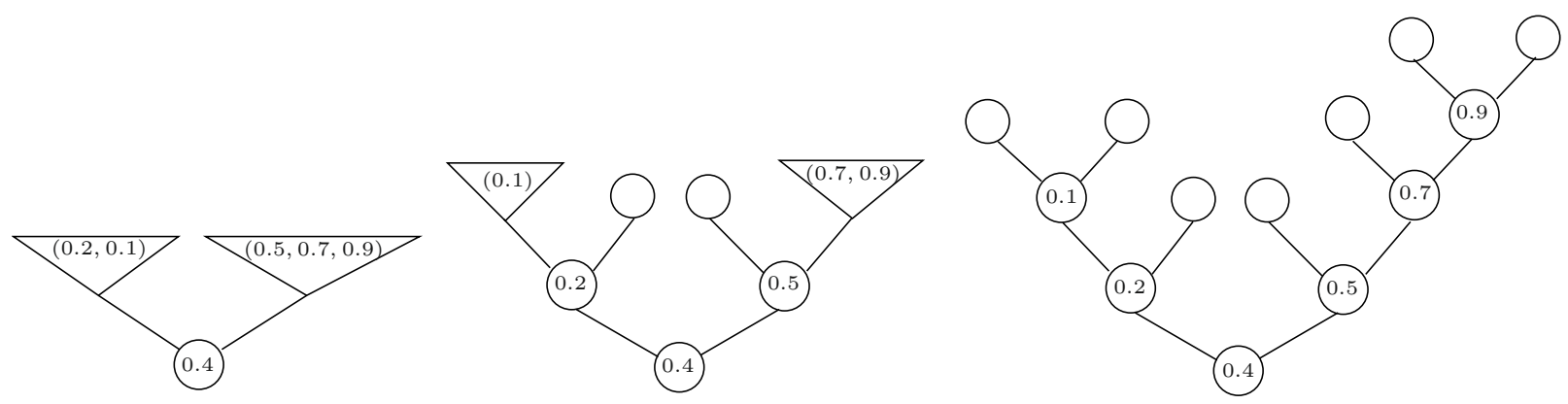

FIgure 1. First, second and last steps of the construction of the labeled BST associated with $(0.4,0.2,0.5,0.7,0.1,0.6)$. The associated BST is obtained by deleting the labels on the last picture.

than $U_{1}$. The labeled BST associated with an empty list is reduced to a leaf. See Figure 1 for an example. This construction induces a law on $\mathcal{B}_{n}:=\{\mathcal{T} \in \mathcal{B}, \# \mathcal{T}=2 n+1\}$, the trees where the labels are deleted (a complete binary tree with $n$ internal nodes always has $n+1$ external nodes).

This BST model can also be obtained by a sequential construction without the $U_{i}$ 's: $\mathcal{T}_{0}$ is reduced to a leaf. Assume $\mathcal{T}_{n}$ is a BST with $n+1$ leaves, then we construct $\mathcal{T}_{n+1}$ by choosing equally likely one of the $n+1$ leaves of $\mathcal{T}_{n}$ and by replacing it by an internal node. The replacement creates two new leaves.

Many properties of BSTs, like the height or the profile, have been studied in the literature: we refer to Mahmoud [17], Chauvin et al. [8], Drmota [10] and Devroye and Hwang [9] and references therein for an overview of the subject.

\subsection{The increments model}

We endow the elements of $\mathbb{U}^{b}$ with random variables, that is we define $\left(c_{u}\right)_{u \in \mathbb{U}^{b}}$ a family of independent random variables on $\mathbb{R}^{2} ; c_{u}:=\left(X_{u 0}, X_{u 1}\right)$ may be seen as a variable associated with the two edges from $u$ to its children $\left(X_{u 0}\right.$ can be seen to be associated with the edge $(u, u 0)$ and $X_{u 1}$ with $\left.(u, u 1)\right) ; X_{\varnothing}:=0$. The $X_{u}$ will be taken as the increments of our branching random walk. We assume the two families $\left(U_{i}\right)$ and $\left(c_{u}\right)$ to be independent. Moreover, we first assume the marginal distributions of all the $c_{u}$ (that is the laws of $X_{u 0}$ and $\left.X_{u 1}\right)$ to be equal. Notice that the variables $X_{u 0}$ and $X_{u 1}$ are not necessarily independent.

Following the above description we construct $\mathcal{T}_{n}$, the BST associated with $\left(U_{i}\right)_{i=1 \cdots n}$, and with each $u \in \mathcal{T}_{n}$ we associate a trajectory of the killed random walk $Y_{u}=\left(Y_{u}(j)\right)_{j \in\{0, \cdots,|u|\}}$ defined by

$$
Y_{u}(0)=0, \quad Y_{u}(j)=\sum_{\substack{v \ll u ; \\|v| \leq j}} X_{v} \quad \forall j \in\{1, \cdots,|u|\}
$$

Each $Y_{u}(j)$ is the sum of the $j$ 's increments associated with the $j$ 's first edges of the path from the root to $u$; with the above assumptions on the $c_{u}$ 's, $Y_{u}(j)$ is a sum of i.i.d. random variables. The finite branching random walk with underlying tree $\mathcal{T}$ is the family of all the trajectories $Y_{u}$, that is $\left(Y_{u}, u \in \mathcal{T}\right)$. See Figure 2 for an example.

We are interested in the occupation measure $\sum \delta_{Y_{u}(|u|)}$ of these random walks, suitably rescaled.

Knowing the height of the node $u$, the variable $Y_{u}(|u|)$ is a sum of i.i.d. random variables, and thus the asymptotics behavior of $Y_{u}(|u|)$, when $|u|$ is large, depends on the stable law, whenever it exists, that contains the distribution of the marginals of the $c_{u}$ 's in its domain of attraction. 

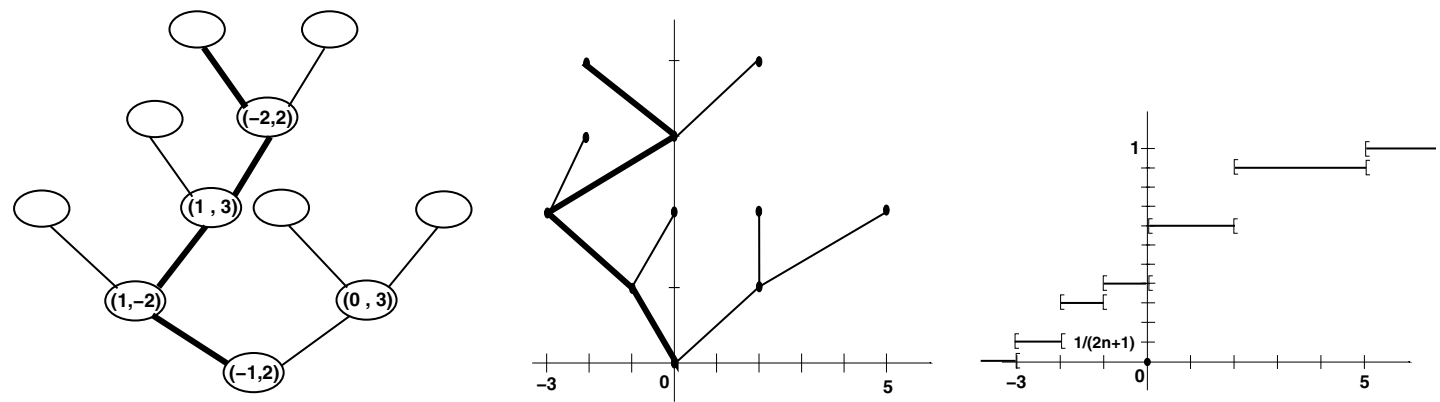

FIGURE 2. BST with the associated $\left(c_{u}\right)$, branching random walk and cumulative function associated with the empirical measure.

\section{Convergence of the occupation measure}

\subsection{The identically distributed case}

Let $\nu$ be a distribution on $\mathbb{R}$ with finite mean $m_{\nu} \in \mathbb{R}^{+} \cup\{+\infty\}$ and variance $\sigma_{\nu}^{2} \in \mathbb{R}^{+} \cup\{+\infty\}$. We assume that $\nu$ belongs to the domain of attraction of a non-degenerate stable law $\mu$ with characteristic exponent $\alpha \in(0,2] \backslash\{1\}$ (for sake of simplicity we restrict our study to the case $\alpha \neq 1$ ), which means that there exist two deterministic sequences $\left(a_{n}\right)$ and $\left(b_{n}\right)$ such that

$$
\frac{\left(\sum_{i=1}^{n} X_{i}\right)-b_{n}}{a_{n}} \underset{n \rightarrow \infty}{\stackrel{\mathcal{D}}{\longrightarrow}} \mu
$$

for any family $\left(X_{i}\right)$ of i.i.d. random variables with law $\nu$. The characteristic function of any stable law $\mu$ is of the form

$$
\phi(t)=\exp \left\{i \gamma t-c|t|^{\alpha}\left[1+i \beta \frac{t}{|t|} \tan \left(\frac{\pi}{2} \alpha\right)\right]\right\}
$$

where $\alpha \in(0,2] \backslash\{1\}, \gamma \in \mathbb{R},|\beta| \leq 1$ and $c \geq 0$. Moreover

- The sequence $\left(a_{n}\right)$ is regularly varying of index $1 / \alpha$, that is

$$
a_{n}=L_{n} \times n^{1 / \alpha}
$$

where $\left(L_{n}\right)$ is a nonnegative sequence satisfying $L_{\lfloor n t\rfloor} / L_{n} \underset{n \rightarrow \infty}{\longrightarrow} 1$, for every $t>0\left(\left(L_{n}\right)\right.$ is slowly varying).

- The sequence $\left(b_{n}\right)$ will be taken as $b_{n}=n m_{\nu}^{\prime}$, where $m_{\nu}^{\prime}=m_{\nu}$ if $\alpha>1$ and $m_{\nu}^{\prime}=0$ if $\alpha<1$ (this is possible, see the proof of Thm. 9.34 of [5]).

When $b_{n}$ is fixed, there exist several choices for $\left(\left(a_{n}\right), \mu\right)$ in order to get $(2.1)$ but, given $\nu$, all non-degenerate $\mu$ have the same characteristic exponent $\alpha$.

- If $\alpha=2, \nu$ is in the domain of attraction of the normal distribution. For $b_{n}=n m_{\nu}, \mu$ can be shown to be centered (using the comparison between $\left(\left(\sum_{i=1}^{n} X_{i}\right)-b_{n}\right) / a_{n}+\left(\left(\sum_{i=1}^{n} \tilde{X}_{i}\right)-b_{n}\right) / a_{n}$ and $\left.\left(\left(\sum_{i=1}^{2 n} X_{i}\right)-b_{2 n}\right) / a_{2 n}\right)$.

- If $\sigma_{\nu}^{2}<+\infty$ we take $a_{n}=\sqrt{n}$ (that is $L_{n} \equiv 1$ ) and thus $\mu$ is $\mathcal{N}\left(0, \sigma_{\nu}^{2}\right)$, according to the central limit theorem.

- If $\sigma_{\nu}^{2}=+\infty$ then $a_{n}=L_{n} \sqrt{n}$ for some $L_{n} \rightarrow+\infty$ (as one can check in the proof of [13] Thm. 5.17).

- If $\alpha \in(0,2) \backslash\{1\}$, the choice of $\left(a_{n}\right)$ determines $\mu$. 
From now on, we assume $\left(a_{n}\right),\left(b_{n}\right)$, and $\mu$ are fixed.

Let $\mathcal{T}_{n}$ be a BST. Taking $u$ uniformly at random in $\mathcal{T}_{n}$ or $\mathcal{T}_{n} \backslash \partial \mathcal{T}_{n}$, the value $|u|$ is "close" to $2 \log n$ (see (4.1) and [6]). Thus, if we assume that both marginals of the $c_{u}$ 's equal distribution $\nu$, this incites us to set

$$
\alpha_{n}:=a_{2 \log n}=L_{2 \log n} \times(2 \log n)^{1 / \alpha} \quad \text { and } \quad \beta_{n}:=b_{2 \log n}=2 m_{\nu}^{\prime} \log n
$$

as normalizing constants in the internal and global occupation measures of the branching random walks defined by

$$
\mu_{n}=\frac{1}{n} \sum_{u \in \mathcal{T}_{n} \backslash \partial \mathcal{T}_{n}} \frac{\delta_{\underline{Y_{u}(|u|)-\beta_{n}}}}{\alpha_{n}} \text { and } \quad \bar{\mu}_{n}=\frac{1}{2 n+1} \sum_{u \in \mathcal{T}_{n}} \frac{\delta_{\underline{Y_{u}(|u|)-\beta_{n}}}}{\alpha_{n}} .
$$

Theorem 2.1. If the marginals of the $c_{u}$ 's belong to the domain of attraction of $\mu$, then

if $\alpha \in(0,2) \backslash\{1\}$ or if $\alpha=2$ and $\sigma_{\nu}^{2}=+\infty$ we have

$$
\mu_{n} \underset{n \rightarrow \infty}{\stackrel{p r o b a}{\longrightarrow}} \mu \quad \text { and } \quad \bar{\mu}_{n} \underset{n \rightarrow \infty}{\stackrel{\text { proba }}{\longrightarrow}} \mu,
$$

and if $\alpha=2$ and $\sigma_{\nu}^{2}<+\infty$ we have

$$
\mu_{n} \underset{n \rightarrow \infty}{\stackrel{\text { proba }}{\longrightarrow}} \mathcal{N}\left(0, \sigma_{\nu}^{2}+m_{\nu}^{2}\right) \quad \text { and } \quad \bar{\mu}_{n} \underset{n \rightarrow \infty}{\stackrel{\text { proba }}{\longrightarrow}} \mathcal{N}\left(0, \sigma_{\nu}^{2}+m_{\nu}^{2}\right),
$$

in the set of probability measures on $\mathbb{R}$ endowed with the weak topology.

In the following, we denote by $\mu_{\infty, \nu}$ the limit measure. Notice that $\mu_{\infty, \nu}$ is always deterministic and that its characteristic exponent is the same as that of $\mu$. The proof of Theorem 2.1 is relegated to Section 4.

\subsection{The non-identically distributed case}

We are now going to show that Theorem 2.1 holds true even if the marginals of the $c_{u}$ 's are not the same. Let $\Lambda^{\star}$ be a distribution on $\mathbb{R}^{2}$ and $\nu_{0}$ and $\nu_{1}$ its two marginals. We assume that $\nu_{0}$ and $\nu_{1}$ are different. We denote by $\Lambda$ the distribution on $\mathbb{R}^{2}$ defined by

$$
\Lambda:=\frac{1}{2}\left(\Lambda^{\star}+\Lambda^{\prime}\right)
$$

where $\Lambda^{\prime}$ is the image of $\Lambda^{\star}$ by $(x, y) \mapsto(y, x)$. Let $Y^{\star}$ and $Y$ be the two branching random walks with underlying tree $\mathcal{T}_{n}$ and with increments $c_{u}^{\star}$ and $c_{u}$ having distribution $\Lambda^{\star}$ and $\Lambda$. The construction of $Y$ can be seen as follows: for any $u$ in $\mathcal{T}_{n}$, we toss a fair coin, if it is tail then $c_{u}$ is distributed as $\Lambda^{\star}$ and if it is head then $c_{u}$ is distributed as $\Lambda^{\prime}$.

Proposition 2.1. Let $\mu_{n}^{\star}, \bar{\mu}_{n}^{\star}, \mu_{n}$ and $\bar{\mu}_{n}$ be the internal and global occupation measures of $Y^{\star}$ and $Y$. We have

$$
\mu_{n} \stackrel{\mathcal{D}}{=} \mu_{n}^{\star} \quad \text { and } \quad \bar{\mu}_{n} \stackrel{\mathcal{D}}{=} \bar{\mu}_{n}^{\star} .
$$

Proposition 2.1 is an analogue of Proposition 7 in Marckert [20]. We give here the main arguments of his proof: any binary tree $\mathcal{T}_{n-1}$ marked with the $c_{u}$ 's corresponds, via a composition of transpositions, to a unique marked tree obtained with the $c_{u}^{\star}$ 's (the transposition around a node $u$ is the map that exchanges the two subtrees rooted in $u$ ). Two marked trees are said to be in the same equivalence class if and only if there exists a composition of transpositions that associates one tree with the other one. Each class contains $2^{n-1}$ trees and one exactly is marked with the $c_{u}^{\star}$ 's. Moreover each binary tree $\mathcal{T}_{n-1}$ can be marked with the $c_{u}$ 's in $2^{n-1}$ ways. Since the underlying trees of a same class are equally likely BSTs and since the occupation measure is invariant by transposition, the result (2.3) holds true. 
As a consequence of Proposition 2.1, the two rescaled occupation measures have the same limit (if any) and thus we have the following result on the convergence of the occupation measure of the branching random walk $Y^{\star}$.

Corollary 2.1. If the marginal distributions of $\Lambda$, defined in (2.2), belong to the domain of attraction of $\mu$, then

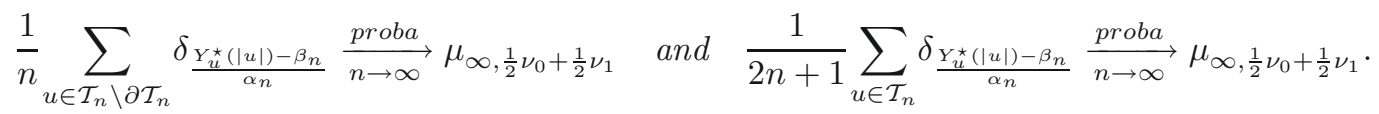

in the set of probability measures endowed with the weak topology, where $\alpha_{n}$ and $\beta_{n}$ are given in Section 2.

Remark 2.1. Using Corollary 2.1 for the case when $c_{u}^{\star}=(-1,+1)$ a.s., we get

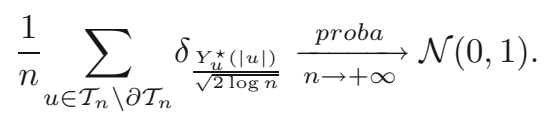

Let $u_{n}$ be a random node uniformly chosen in $\mathcal{T}_{n} \backslash \partial \mathcal{T}_{n}$, as a consequence of (2.4) we have

$$
\frac{Y_{u_{n}}^{\star}\left(\left|u_{n}\right|\right)}{\sqrt{2 \log n}} \underset{n \rightarrow+\infty}{\stackrel{\mathcal{D}}{\longrightarrow}} \mathcal{N}(0,1) .
$$

This particular case of $c_{u}$ 's have been recently studied by Kuba and Panholzer [15]; they show, among other things, the above convergence. They also prove that the rate of convergence is of order $\mathcal{O}(1 / \sqrt{\log n})$.

Sections 3.1 and 3.2 handle the study of the behavior of a random process named homogeneous fragmentation and of the profile of another type of random trees named the recursive trees. The results we obtain on both processes are direct consequences of Theorem 2.1 and Corollary 2.1.

\section{Applications}

\subsection{Empirical measure of homogeneous fragmentations}

Here we are interested in homogeneous fragmentations with no erosion, no loss of mass and where a fragment is always divided, at random, into two parts. The homogeneous fragmentations (and more general fragmentations) have been studied a lot these last years, particularly by Bertoin (see e.g. [2]). Following the steps in Chauvin et al. [8], we give a definition of the fragmentation process $(\mathcal{F}(t))_{t \geq 0}$ of the interval $(0,1)$ we are interested in. This model contains not only the state at time $t$ but also the history of the process. Let $\mathcal{F}(0):=(0,1)$ and $t_{1} \sim \operatorname{Exp}(1)$, an exponential random variable, associated with $\mathcal{F}(0)$, seen as the lifetime of the interval. At time $t_{1}$, the process $\mathcal{F}$ jumps, the interval $\mathcal{F}(0)$ splits into two parts $I_{0}$ and $I_{1}$ such that the size of the left fragment is given by a random variable $Z$ having distribution $\nu_{0}$ on $(0,1): \mathcal{F}\left(t_{1}\right):=\left(I_{0}, I_{1}\right)=((0, Z),(Z, 1))$. We denote by $\nu_{1}$ the law of $1-Z$, the length of $I_{1}$. After each jump time the fragments of $\mathcal{F}$ behave independently of each other and each fragment splits after an $\operatorname{Exp}(1)$ distributed lifetime into two parts. When $I_{u}$ splits, we denote by $I_{u 0}$ and $I_{u 1}$ the left and right fragments and we call them the children of $I_{u}$. Conditionally on $I_{u}$, the length of the left interval, denoted by $\left|I_{u 0}\right|$, is $\left|I_{u}\right| Z_{u}$ where $Z_{u}$ is an independent copy of $Z$. Thanks to the lack of memory of the exponential distribution, when $n$ fragments are present, each of them will split first equally likely. Of course the behavior of the process depends on $\nu_{0}$ and $\nu_{1}$, the laws of the dependent variables $Z_{u}$ and $1-Z_{u}$. We call this fragmentation an homogeneous fragmentation with law $\nu_{0}$.

In this construction, the fragments are naturally indexed by the leaves of a binary tree that encodes the history of the fragmentation. More formally, a continuous binary tree process $\left(\mathbb{T}_{t}\right)_{t \geq 0}$ on $\mathcal{B}$ can be associated with $(\mathcal{F}(t))_{t \geq 0}$. We define $\mathbb{T}_{t}$ by the set of its leaves

$$
\partial \mathbb{T}_{t}:=\left\{u, I_{u} \in \mathcal{F}(t)\right\}
$$


Let $t_{n}$ be the $n$th jump time of the processes $\left(\mathbb{T}_{t}\right)$ and $(\mathcal{F}(t))$. Thanks to [8] we have the following results:

$$
\frac{t_{n}}{\log n} \stackrel{n \rightarrow+\infty}{\longrightarrow} 1 \text { a.s. }
$$

and, for any $n \geq 1, \mathbb{T}_{t_{n}}$ is a BST of size $n$.

The size of the fragments in this fragmentation process can be described as an homogeneous fragmentation with the formalism of Bertoin, that is giving the dislocation measure $\nu_{d}$ on $\mathcal{S}:=\left\{\left(x_{1}, x_{2}, \ldots\right) ;\right.$ s.t. $x_{1} \geq x_{2}$ $\left.\geq \ldots \geq 0, \sum x_{i}=1\right\}$. Here, the measure $\nu_{d}$ is defined by

$$
\int_{\mathcal{S}} f(\mathbf{x}) \nu_{d}(\mathrm{~d} \mathbf{x})=\int_{0}^{1} f(s \vee(1-s), s \wedge(1-s), 0, \ldots) \nu_{0}(\mathrm{~d} s),
$$

for every non-negative measurable $f$.

We are interested in the empirical measure of the logarithm of the size of the fragments of $\mathcal{F}$ defined by

$$
f_{t}:=\sum_{u \in \partial \mathbb{T}(t)} \delta_{-\log \left|I_{u}\right|} .
$$

We thus have

$$
f_{t_{n}}=\sum_{u \in \partial \mathbb{T}_{t_{n}}} \delta_{Y_{u}(|u|)},
$$

where $Y$ is the branching random walk on the binary search tree $\mathbb{T}_{t_{n}}$, with increments having the following distribution: for any $u \in \mathbb{T}_{t_{n}}, X_{u 0}$ is distributed as $-\log \left(Z_{u}\right)$ and $X_{u 1}$ is distributed as $-\log \left(1-Z_{u}\right)$, where $\left(Z_{u}\right)$ is a family of i.i.d. r.v. having law $\nu_{0}$. We denote by $\hat{\nu}_{0}$ and $\hat{\nu}_{1}$ the two distributions of the increments.

The study of fragmentation processes is concerned with the behavior of the fragment sizes. In [2], Bertoin gives a result on $\sum\left|I_{u}\right| \delta_{-\log \left|I_{u}\right|}$, the empirical measure of the logarithm of the size of the fragments balanced by their own size. He shows, under some assumptions on the dislocation measure of homogeneous fragmentations, that the rescaled measure converges to a Gaussian law. Here we show, in a more general non-Gaussian case, that the simple occupation measure $f_{t_{n}}$, suitably rescaled, converges weakly to a deterministic measure (as the limiting distribution of Bertoin). The following result is a direct consequence of Corollary 2.1, used for the external occupation measure.

Theorem 3.1. Let $(\mathcal{F}(t))$ be an homogeneous fragmentation with law $\nu_{0}$. If $\frac{1}{2} \hat{\nu}_{0}+\frac{1}{2} \hat{\nu}_{1}$ belongs to the domain of attraction of $\mu$, then

$$
\frac{1}{n+1} \sum_{I_{u} \in \mathcal{F}\left(t_{n}\right)} \delta_{\frac{-\log \left|I_{u}\right|-\beta_{n}}{\alpha_{n}}}^{\stackrel{\text { proba }}{n \rightarrow+\infty}} \mu_{\infty, \frac{1}{2} \hat{\nu}_{0}+\frac{1}{2} \hat{\nu}_{1}}
$$

in the set of probability measure on $\mathbb{R}$ endowed with the weak topology, where $\alpha_{n}$ and $\beta_{n}$ are given in Section 2.

\subsection{Profile of recursive trees}

A recursive tree process is a random sequence $\left(\mathfrak{T}_{n}, n \geq 1\right)$ of plane trees, where $\mathfrak{T}_{n}$ has $n$ nodes, built recursively under the following dynamics: $\mathfrak{T}_{1}$ is reduced to a simple node; the tree $\mathfrak{T}_{n+1}$ is obtained from $\mathfrak{T}_{n}$ by adjoining a child to one of the nodes. The choice is uniform over the nodes and the new node is inserted as the rightmost son. Notice that $\mathfrak{T}_{2}$ is deterministic and that, for $n \geq 3$, recursive trees of size $n$ are not equally likely. Let $\tilde{\mathbb{P}}_{n}$ be the law on $\mathcal{P}_{n}:=\{\mathfrak{T} \in \mathcal{P}, \# \mathfrak{T}=n\}$ induced by this construction (that is a recursive tree of size $n$ is a random element of $\mathcal{P}_{n}$ under $\tilde{\mathbb{P}}_{n}$ ). Figure 3 shows two recursive trees of size 4 with the possible ways to construct them.

This process of plane trees has been first proposed to study the spread of epidemics (Moon, 1974) and many parameters on that trees have been studied: the height, the depth of nodes, the path length, the maximal degree... (see e.g. Smythe and Mahmoud [19]). 


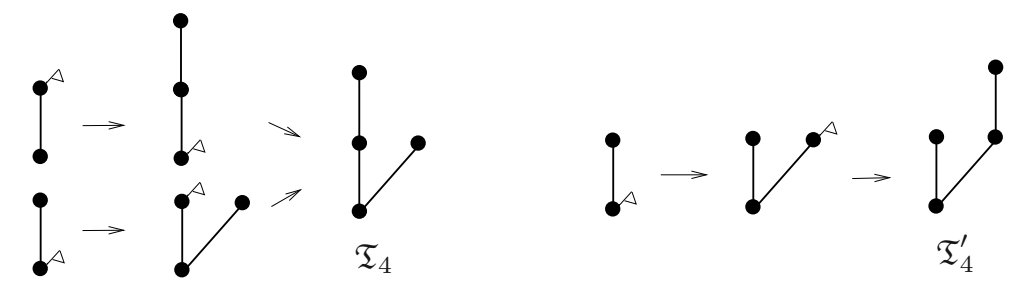

Figure 3. Two possible recursive trees of size 4 with the different ways to obtain them (the flags indicate where the nodes are inserted). Since $\mathfrak{T}_{4}$ is obtained from two different recursive trees of size 3 , unlike $\mathfrak{T}_{4}^{\prime}$, we have $\tilde{\mathbb{P}}_{4}\left(\mathfrak{T}_{4}\right)=\frac{1}{3}$ and $\tilde{\mathbb{P}}_{4}\left(\mathfrak{T}_{4}^{\prime}\right)=\frac{1}{6}$.

We are interested in $X_{n}(k)$, the number of nodes in the recursive tree $\mathfrak{T}_{n}$ at level $k$. Let $\left(p_{n}(k)\right)_{k}$ be the empirical distribution function of the profile of $\mathfrak{T}_{n}$ defined by

$$
p_{n}(k):=\sum_{i=1}^{k} \frac{X_{n}(i)}{n} .
$$

We define the rescaled cumulated profile $q_{n}$ by

$$
q_{n}(\lambda):=p_{n}(\lfloor\log n+\lambda \sqrt{2 \log n}\rfloor), \quad \forall \lambda \in \mathbb{R} .
$$

We have the following theorem.

\section{Theorem 3.2.}

$$
\left(q_{n}(\lambda)\right)_{\lambda \in \mathbb{R}} \underset{n \rightarrow+\infty}{\stackrel{\text { proba }}{\longrightarrow}}(\mathbb{P}(N \leq \lambda))_{\lambda \in \mathbb{R}}
$$

for the Skorohod topology, where $N$ is a random variable with law $\mathcal{N}\left(0, \frac{1}{2}\right)$.

The proof of Theorem 3.2 is relegated to Section 4.2.

Recently Fuchs et al. [11] have studied the profile of recursive trees; using the contraction method and the method of moments they proved that $X_{n}(k)$, normalized by its mean, converges in distribution to a random variable which is the fixed point of an explicit equation (their results are of a different nature from Thm. 3.2).

\section{Proofs}

\subsection{Proof of Theorem 2.1}

The proof of Theorem 2.1 we detail below is a consequence of the convergences of the mean and of the variance of the empirical distribution function associated with $\mu_{n}$ (see Lems. 4.1 and 4.2). This approach is different from Janson and Marckert [12]'s one to show the convergence of the occupation measure of a branching random walk on conditioned Galton-Watson (GW) trees to ISE, where the fact that normalized GW trees have a limit (the Aldous continuum random tree) is crucial. There is no similar result for BSTs in the literature.

In this section, we first prove the convergence of $\mu_{n}$. For the convergence of $\bar{\mu}_{n}$, the proof runs along the same lines, see Remark 4.1 for some details.

Lemma 4.1. Under the assumptions of Theorem 2.1, we have $\forall x \in \mathbb{R}$

$$
\mathbb{E}\left(\mu_{n}((-\infty, x]) \underset{n \rightarrow \infty}{\longrightarrow} \mu_{\infty}((-\infty, x]) .\right.
$$


Proof. Let $u_{n}$ be chosen equally likely among the nodes of $\mathcal{T}_{n} \backslash \partial \mathcal{T}_{n}$. We get the equalities

$$
\begin{aligned}
\mathbb{E}\left(\mu_{n}((-\infty, x])\right. & =\mathbb{E}\left(\frac{1}{n} \times \sum_{u \in \mathcal{T}_{n} \backslash \partial \mathcal{T}_{n}} \delta_{\underline{Y_{u}(|u|)-\beta_{n}}}((-\infty, x])\right) \\
& =\mathbb{E}\left(\mathbb{1}_{(-\infty, x]}\left(\frac{Y_{u_{n}}\left(\left|u_{n}\right|\right)-\beta_{n}}{\alpha_{n}}\right)\right) .
\end{aligned}
$$

By (1.2), this is equal to $C_{n}:=\mathbb{P}\left(\left(\left(\sum_{v \ll u_{n}} X_{v}\right)-\beta_{n}\right) / \alpha_{n} \leq x\right)$.

The sum contains $\left|u_{n}\right|$ terms. In terms of quicksort the random variable $\left|u_{n}\right|$ is the cost of a successful search in a labeled binary search tree of size $n$. Louchard [16] shows that

$$
\frac{\left|u_{n}\right|-2 \log (n)}{\sqrt{2 \log (n)}} \stackrel{\mathcal{D}}{\longrightarrow} \mathcal{N}(0,1) .
$$

Let $H_{\varepsilon, n}$ be the event $\left\{|| u_{n}|-2 \log (n)| \leq(2 \log (n))^{\frac{1}{2}+\varepsilon}\right\}$, with $\left.\varepsilon \in\right] 0, \frac{1}{2}\left[\right.$. Since $\mathbb{P}\left(H_{\varepsilon, n}\right)$ goes to 1 , by $(4.1), C_{n}$ and $\mathbb{P}\left(\left(\left(\sum_{v \ll u_{n}} X_{v}\right)-\beta_{n}\right) / \alpha_{n} \leq x \mid H_{\varepsilon, n}\right)$ have the same limit (if any).

The sum $\left(\sum_{v \ll u_{n}} X_{v}\right)-\beta_{n}$ has the following representation

$$
\sum_{i=1}^{d_{n}}\left(X_{i}-m_{\nu}^{\prime}\right)+\sum_{i=1}^{\left|u_{n}\right|-d_{n}}\left(\tilde{X}_{i}-m_{\nu}^{\prime}\right)+m_{\nu}^{\prime}\left(\left|u_{n}\right|-2 \log n\right),
$$

where $d_{n}=2 \log n-(2 \log n)^{\frac{1}{2}+\varepsilon}$ and $\left(X_{i}\right)$ and $\left(\tilde{X}_{i}\right)$ are two independent families of i.i.d. random variables with common law $\nu$. Recall that $m_{\nu}^{\prime}$ is the mean of $\nu$ if it is finite, and zero otherwise. To end the proof of Lemma 4.1 we study the asymptotics of the three terms in (4.2).

- The term $\sum_{i=1}^{d_{n}}\left(X_{i}-m_{\nu}^{\prime}\right) / \alpha_{n}$ converges in distribution to the stable law $\mu$ (recall that $\mu=\mathcal{N}\left(0, \sigma_{\nu}^{2}\right)$ when $\alpha=2$ and $\left.\sigma^{2}<+\infty\right)$.

- It is known (see Thm. 16.14 of [13]) that (2.1) implies the convergence of the process $\left(\sum_{i=1}^{\left\lfloor e_{n} t\right\rfloor}\left(\tilde{X}_{i}-m_{\nu}^{\prime}\right) /\left(f_{n} t\right)\right.$, $t \geq 0)$ in the Skorohod space, to a stable Levy process of index $\alpha$, if $f_{n} \sim L_{n} \times \mathrm{e}_{n}^{1 / \alpha}$ with $e_{n} \rightarrow+\infty$ and $\left(L_{n}\right)$ is slowly varying. Thus

$$
\left(\sum_{i=1}^{\left\lfloor c_{n} t\right\rfloor}\left(\tilde{X}_{i}-m_{\nu}^{\prime}\right) / \alpha_{n}, t \geq 0\right) \underset{n \rightarrow \infty}{\stackrel{\mathcal{D}}{\longrightarrow}} 0,
$$

where $c_{n}:=2(2 \log n)^{\frac{1}{2}+\varepsilon}$. Since, if $X_{n} \rightarrow X$ for the Skorohod topology then $\max \left(X_{n}\right) \rightarrow \max (X)$ in distribution, we get

$$
\max _{1 \leq k \leq c_{n}} \sum_{i=1}^{k}\left(\tilde{X}_{i}-m_{\nu}^{\prime}\right) / \alpha_{n} \underset{n \rightarrow \infty}{\stackrel{\mathcal{D}}{\longrightarrow}} 0
$$

On $H_{\varepsilon, n},\left|u_{n}\right|-d_{n}$ is smaller than $c_{n}$. Hence, we have shown that $\sum_{i=1}^{\left|u_{n}\right|-d_{n}}\left(\tilde{X}_{i}-m_{\nu}^{\prime}\right) / \alpha_{n}$ goes to zero in probability. 
- For the third term in (4.2) normalized by $\alpha_{n}$, when $\sigma_{\nu}^{2}<+\infty$ we have $\alpha_{n}=\sqrt{2 \log n}$ and $m_{\nu}^{\prime}=m_{\nu}$. Thus, by (4.1)

$$
m_{\nu}^{\prime} \frac{\left|u_{n}\right|-2 \log n}{\alpha_{n}} \underset{n \rightarrow \infty}{\stackrel{\mathcal{D}}{\longrightarrow}} \mathcal{N}\left(0, m_{\nu}^{2}\right) .
$$

When $\alpha \in(0,2) \backslash\{1\}$ or when $\alpha=2$ and $\sigma_{\nu}^{2}=+\infty$ (recall that in this case $L_{n} \rightarrow+\infty$ ), we have $\alpha_{n} / \sqrt{2 \log n} \rightarrow$ $+\infty$, and then, by (4.1)

$$
m_{\nu}^{\prime} \frac{\left|u_{n}\right|-2 \log n}{\alpha_{n}} \underset{n \rightarrow \infty}{\stackrel{p r o b a}{\longrightarrow}} \quad 0
$$

Now, since the extremal terms in (4.2) are independent and the middle one vanishes in probability, the result follows by using, for example, Theorem 25.4 of [4] (that is, if $X_{n} \stackrel{\mathcal{D}}{\longrightarrow} X$ and $Y_{n} \stackrel{\text { proba }}{\longrightarrow} 0$ then $X_{n}+Y_{n} \stackrel{\mathcal{D}}{\longrightarrow} X$ ).

In (4.2), the two terms that contribute to the limit are the first and the third one (corresponding to a centered version of the law, and to a corrected term due to the mean of the increments). When $\sigma_{\nu}^{2}<+\infty$, these two terms have the same order. When $\sigma_{\nu}^{2}=+\infty$ the first one dominates.

Lemma 4.2. Under the assumptions of Theorem 2.1, we have $\forall x \in \mathbb{R}$

$$
\mathbb{V}\left(\mu_{n}((-\infty, x])\right) \underset{n \rightarrow \infty}{\longrightarrow} 0 .
$$

Proof. Let $u_{n}$ and $v_{n}$ be two nodes uniformly and independently chosen on $\mathcal{T}_{n} \backslash \partial \mathcal{T}_{n}$. We get for any $x \in \mathbb{R}$ and any $n \in \mathbb{N}$

$$
\begin{aligned}
\mathbb{E}\left(\mu_{n}((-\infty, x])\right)^{2} & =\mathbb{E}\left(\mathbb{1}_{(-\infty, x]}\left(\frac{Y_{u_{n}}\left(\left|u_{n}\right|\right)-\beta_{n}}{\alpha_{n}}\right) \mathbb{1}_{(-\infty, x]}\left(\frac{Y_{v_{n}}\left(\left|v_{n}\right|\right)-\beta_{n}}{\alpha_{n}}\right)\right) \\
& =\mathbb{P}\left(\frac{Y_{u_{n}}\left(\left|u_{n}\right|\right)-\beta_{n}}{\alpha_{n}} \leq x, \frac{Y_{v_{n}}\left(\left|v_{n}\right|\right)-\beta_{n}}{\alpha_{n}} \leq x\right) .
\end{aligned}
$$

Let $u_{n} \wedge v_{n}$ be the deepest common ancestor of $u_{n}$ and $v_{n}$. To compute the limit in (4.3) we investigate the dependence between $Y_{u_{n}}\left(\left|u_{n}\right|\right)$ and $Y_{v_{n}}\left(\left|v_{n}\right|\right)$. This dependence comes from the values of the ancestors of $u_{n} \wedge v_{n}$ which contribute both to $Y_{u_{n}}\left(\left|u_{n}\right|\right)$ and to $Y_{v_{n}}\left(\left|v_{n}\right|\right)$ and can also come from the values of the children of $u_{n} \wedge v_{n}$.

Let $\Delta_{u_{n}, v_{n}}$ be the path distance between $u_{n}$ and $v_{n}$ in $\mathcal{T}_{n}$. Mahmoud and Neininger [18] have shown

$$
\frac{\Delta_{u_{n}, v_{n}}-4 \log (n)}{\sqrt{4 \log (n)}} \underset{n \rightarrow \infty}{\stackrel{\mathcal{D}}{\longrightarrow}} \mathcal{N}(0,1) .
$$

Using the relation $\Delta_{u_{n}, v_{n}}=\left|u_{n}\right|+\left|v_{n}\right|-2\left|u_{n} \wedge v_{n}\right|$, (4.4) and (4.1) one gets, for any $\varepsilon>0$,

$$
\frac{\left|u_{n} \wedge v_{n}\right|}{(2 \log (n))^{\frac{1}{2}+\varepsilon}} \underset{n \rightarrow \infty}{\stackrel{\text { proba }}{\longrightarrow}} 0 .
$$

In order to get the limit in (4.3) we write

$$
Y_{u_{n}}\left(\left|u_{n}\right|\right) \stackrel{\mathcal{D}}{=} \tilde{X}_{1}+\sum_{i=1}^{\left|u_{n} \wedge v_{n}\right|} X_{i}+\sum_{i=2}^{\left|u_{n}\right|-\left|u_{n} \wedge v_{n}\right|} \tilde{X}_{i}
$$

and

$$
Y_{v_{n}}\left(\left|v_{n}\right|\right) \stackrel{\mathcal{D}}{=} \hat{X}_{1}+\sum_{i=1}^{\left|u_{n} \wedge v_{n}\right|} X_{i}+\sum_{i=2}^{\left|v_{n}\right|-\left|u_{n} \wedge v_{n}\right|} \hat{X}_{i}
$$


where the random variables $\left(X_{i}\right)_{i \geq 1},\left(\tilde{X}_{i}\right)_{i \geq 2}$ and $\left(\hat{X}_{i}\right)_{i \geq 2}$ are all i.i.d. with law $\nu ; \tilde{X}_{1}$ and $\hat{X}_{1}$ are the values attached to the children of $u_{n} \wedge v_{n}$ and may be dependent. Using (4.5) we prove, with the same arguments as those in the proof of Lemma 4.1, that the common sum of (4.6) and (4.7), that is the sum of the $X_{i}$, normalized by $\alpha_{n}$, goes to zero in probability. Moreover we have $\frac{\tilde{X}_{1}}{\alpha_{n}} \underset{n \rightarrow+\infty}{\stackrel{p r o b a}{\longrightarrow}} 0$ and $\frac{\tilde{X}_{1}}{\alpha_{n}} \underset{n \rightarrow+\infty}{\stackrel{p r o b a}{\longrightarrow}} 0$. Whence, using the independence between the remaining sums of (4.6) and (4.7), that is the sum on the $\tilde{X}_{i}$ and the sum on the $\hat{X}_{i}$, we get that the quantity (4.3) has the same limit (if any) as

$$
\mathbb{P}\left(\left(-\beta_{n}+\sum_{i=2}^{\left|u_{n}\right|-\left|u_{n} \wedge v_{n}\right|} \tilde{X}_{i}\right) / \alpha_{n} \leq x\right) \mathbb{P}\left(\left(-\beta_{n}+\sum_{i=2}^{\left|v_{n}\right|-\left|u_{n} \wedge v_{n}\right|} \hat{X}_{i}\right) / \alpha_{n} \leq x\right) .
$$

Using the same methods as in Lemma 4.1, we finally find that $\left.\left.\mathbb{E}\left[\left(\mu_{n}(]-\infty, x\right]\right)\right)^{2}\right]$ has the same limit as $\left.\left.\left[\mathbb{E}\left(\mu_{n}(]-\infty, x\right]\right)\right)\right]^{2}$.

Proof of Theorem 2.1. Thanks to Lemmas 4.1 and 4.2, for any $x \in \mathbb{R}$, the sequence $\left(F_{n}(x)\right)$ converges in probability to $F_{\infty, \nu}(x)$, where $F_{n}$ and $F_{\infty, \nu}$ are respectively the distribution functions of $\mu_{n}$ and of $\mu_{\infty, \nu}\left(F_{\infty, \nu}\right.$ and $\mu_{\infty, \nu}$ are deterministic).

A well known result of analysis is the following: if a sequence of bounded increasing functions, having limits zero at $-\infty$ and one at $+\infty$, converges pointwise to a continuous function then the convergence is uniform. Thus, since the function $F_{\infty, \nu}$ is continuous, we deduce the uniform convergence of $F_{n}$ to $F_{\infty, \nu}$ in probability. This entails the weak convergence of $\mu_{n}$ to $\mu_{\infty, \nu}$ in probability.

Remark 4.1. We proved Theorem 2.1 for the internal occupation measure $\mu_{n}$. To prove the result for $\bar{\mu}_{n}$ it suffices to find similar results to (4.1) and (4.5) for the external nodes. Brown and Shubert [6] have shown that the asymptotics (4.1) is true for $u_{n}$ uniform on $\partial \mathcal{T}_{n}$ and we obtain (4.5) for the leaves using (4.4), a proof runs as follows: let $C_{I}$ and $C_{E}$ be the sets

$$
C_{I}:=\left\{(u, v) \in\left(\mathcal{T}_{n} \backslash \partial \mathcal{T}_{n}\right)^{2} \text { such that }\left|\Delta_{u, v}-4 \log n\right|>(4 \log n)^{\frac{1}{2}+\varepsilon}\right\} .
$$

and

$$
C_{E}:=\left\{(u, v) \in\left(\partial \mathcal{T}_{n}\right)^{2} \text { such that }\left|\Delta_{u, v}-4 \log n\right|>(4 \log n)^{\frac{1}{2}+\varepsilon}+2\right\} .
$$

Using the projection from $C_{E}$ to $C_{I}$, which returns the parent of each leaf, we get $\# C_{E} \leq 4 \times \# C_{I}$. Thus $\# C_{E} / n^{2}$ goes to zero in probability and, using the relation $\Delta_{u, v}=|u|+|v|-2|(u \wedge v)|$ we get that (4.5) holds for $u_{n}$ and $v_{n}$ uniformly and independently chosen on $\partial \mathcal{T}_{n}$.

Moreover, the main result is restricted here to $\alpha \neq 1$, but the symmetric case for $\alpha=1$ also works, since we can take $b_{n} \equiv 0$ for the convergence (2.1).

\subsection{Proof of Theorem 3.2}

Let us see that Theorem 3.2 is a consequence of Corollary 2.1. Following the formalism of Marckert [20], we define a map $\Phi$, named rotation correspondence, from the set $\mathcal{P}$ of plane trees to the set $\mathcal{B}$ of complete binary trees (this is referred to as the natural correspondence in Knuth [14]). With a plane tree $\mathfrak{T}_{n}$ is associated a binary tree $\mathcal{T}_{n-1}$ such that $\Phi\left(\mathfrak{T}_{n}\right)=\mathcal{T}_{n-1}$. It means that to each node $a$ of $\mathfrak{T}_{n}$ corresponds an internal node of $\mathcal{T}_{n-1}$, denoted by $\Phi(a)$, verifying the following three points:

(a) If $a$ is the leftmost son of the root of $\mathfrak{T}_{n}$ then $\Phi(a)=\varnothing$.

(b) $\left(\{a, b\} \subset \mathfrak{T}_{n}, b=a 0\right) \Leftrightarrow\left(\{\Phi(a), \Phi(b)\} \subset \mathcal{T}_{n-1}, \Phi(b)=\Phi(a) 0\right)$.

(c) $\left(\{a, b\} \subset \mathfrak{T}_{n}, a=d c, b=d(c+1)\right) \Leftrightarrow\left(\{\Phi(a), \Phi(b)\} \subset \mathcal{T}_{n-1}, \Phi(b)=\Phi(a) 1\right)$. 

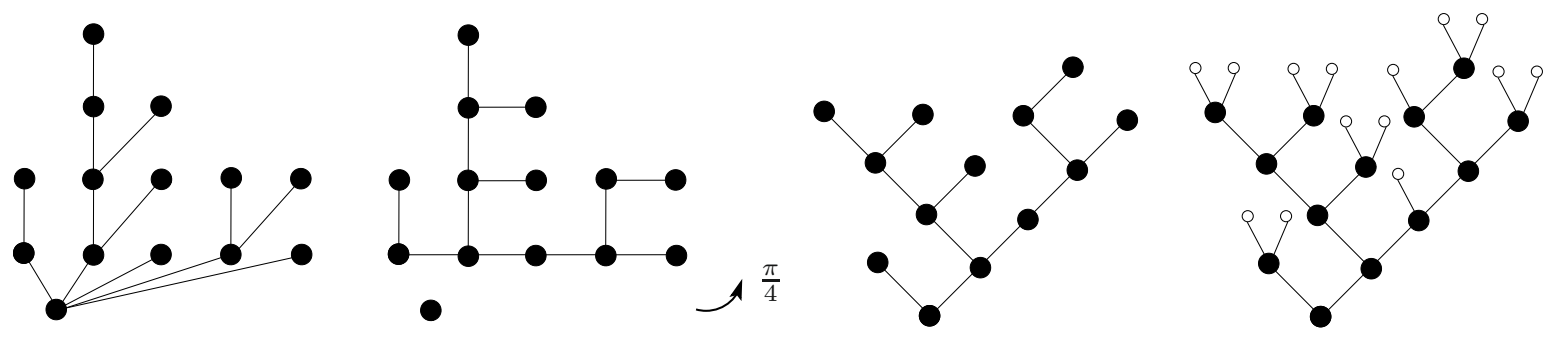

Figure 4. The three steps of the map $\phi$.

$\mathcal{T}_{n-1}$ is obtained by adding the leaves to the internal nodes. (b) means that a relation father-leftmost son in $\mathfrak{T}_{n}$ corresponds to a relation father-left son in $\mathcal{T}_{n-1}$. (c) means that a relation between a node and its first brother on its right in $\mathfrak{T}_{n}$ corresponds to a relation father-right son in $\mathcal{T}_{n-1}$. Figure 4 illustrates why the correspondence is called a rotation. To get $\mathcal{T}_{n-1}$ from $\mathfrak{T}_{n}$, remove the root, keep the edges father-leftmost son and add an edge between each node and its first brother on its right (if any). Make a $\pi / 4$ rotation. This gives the internal nodes of $\mathcal{T}_{n-1}$. Add the leaves to obtain a complete binary tree.

Let $\Psi:=\Phi^{-1}$. The replacement of a leaf by an internal node in a complete binary tree $\mathcal{T}$ results in the addition of a node as a rightmost son in $\Psi(\mathcal{T})$. Thus, since the insertion in both processes is uniform, the random process of complete binary trees $\left(\Phi\left(\mathfrak{T}_{n}\right), n \geq 1\right)$ is a BST process: this implies that both processes $\left(\Phi\left(\mathfrak{T}_{n}\right), n \geq 1\right)$ and $\left(\mathcal{T}_{n}, n \geq 0\right)$ have the same law. Hence the random variables on $\mathcal{P}_{n+1}$ can be seen as random variables on $\mathcal{B}_{n}$ through the map $\Psi$.

From now on we denote by $\mathfrak{T}_{n}$ a recursive tree and by $\mathcal{T}_{n-1}$ the $\operatorname{BST} \Phi\left(\mathfrak{T}_{n}\right)$. For any $u=d_{1} d_{2} \ldots d_{k} \in \mathcal{T}_{n-1}$, we denote by $L(u)$ the left-depth of $u$, that is $L(u)=\#\left\{i \mid d_{i}=0\right\}(L(u)$ is the number of left-steps from the root to $u$ ). By the rotation correspondence the height of a node $a$ in $\mathfrak{T}_{n}-\{\varnothing\}$ minus 1 is equal to the left-depth of $\Phi(a)$ in $\mathcal{T}_{n-1}$. In order to count the left-depth of each internal node, we construct a branching random walk $Y^{\star}$ with underlying tree $\mathcal{T}_{n-1}$ and where the law of the increments is given by

$$
\mathbb{P}\left(c_{u}^{\star}=(1,0)\right)=1, \quad \forall u \in \mathbb{U}^{b} .
$$

With these increments, $Y_{u}^{\star}(|u|)$ is equal to $L(u)$ and is also equal to $|\Psi(u)|-1$. Thus we have

$$
\begin{aligned}
X_{n}(k) & =\#\left\{a \in \mathfrak{T}_{n},|a|=k\right\} \\
& =\#\left\{u \in \mathcal{T}_{n-1}, L(u)=k-1\right\} \\
& =\#\left\{u \in \mathcal{T}_{n-1}, Y_{u}^{\star}(|u|)=k-1\right\} .
\end{aligned}
$$

The occupation measure associated with $Y^{\star}$ is

$$
\mu_{n-1}^{\star}=\frac{1}{n-1} \sum_{u \in \mathcal{T}_{n-1} \backslash \partial \mathcal{T}_{n-1}} \delta_{\frac{Y_{u}^{\star}(|u|)-\log (n-1)}{\sqrt{2 \log (n-1)}}} .
$$

We thus have

$$
p_{n}(k)=\frac{n-1}{n} \mu_{n-1}^{\star}\left(\left(-\infty, \frac{k-1-\log (n-1)}{\sqrt{2 \log (n-1)}}\right]\right) .
$$


Using Corollary 2.1 for the distribution $\Lambda$ given by

$$
\mathbb{P}\left(c_{u}=(1,0)\right)=\mathbb{P}\left(c_{u}=(0,1)\right)=\frac{1}{2}, \quad \forall u \in \mathbb{U}^{b}
$$

we get

$$
\mu_{n-1}^{\star} \underset{n \rightarrow+\infty}{\stackrel{p r o b a}{\longrightarrow}} \mathcal{N}\left(0, \frac{1}{2}\right)
$$

in the set of probability measures for the weak topology, which ends the proof of Theorem 3.2.

\section{Conclusion And Perspectives}

Two important tools of the proof of Theorem 2.1 are the properties of the stable laws and some asymptotics properties of the BSTs. If we consider the branching random walk with another type of underlying trees that still verify (4.1) and (4.5) then an analog of Theorem 2.1 can be proved. It seems to be the case of the recursive trees studied in Section 3.2.

Acknowledgements. J.-F. Marckert is at the origin of this work, I would like to thank him for all his helpful remarks. I also thank one referee for his very efficacious comments.

\section{REFERENCES}

[1] D. Aldous, Tree-based models for random distribution mass. J. Statist. Phys. 73 (1993) 625-641.

[2] J. Bertoin, The asymptotic behavior of fragmentation processes. J. Eur. Math. Soc. 5 (2003) 395-416.

[3] J.D. Biggins, Martingale convergence in the branching random walk. J. Appl. Probab. 14 (1977) 25-37.

[4] P. Billingsley, Probability and measure. Second edition. John Wiley \& Sons, New York (1986).

[5] L. Breiman, Probability. Second edition. SIAM (1992).

[6] G.G. Brown and B.O. Shubert, On random binary trees. Math. Oper. Res. 9 (1985) 43-65.

[7] P. Chassaing and G. Schaeffer, Random planar lattices and integrated superbrownian excursion. Probab. Theory Relat. Fields 128 (2004) 161-212.

[8] B. Chauvin, T. Klein, J.F. Marckert and A. Rouault, Martingales and profile of binary search trees. Electron. J. Probab. 10 (2005) 420-435.

[9] L. Devroye and H.K. Hwang, Width and more of the profile for random trees of logarithmic height. Ann. Appl. Probab. 16 (2006) 886-918.

[10] M. Drmota, Profile and height of random binary search trees. J. Iranian Stat. Soc. 3 (2004) 117-138.

[11] M. Fuchs, H.-K. Hwang and R. Neininger, Profiles of random trees: limit theorems for random recursive trees and binary search trees. Available at: http://algo.stat.sinica.edu.tw (2005).

[12] S. Janson and J.F. Marckert, Convergence of discrete snakes. J. Theory Probab. 18 (2005) 615-645.

[13] O. Kallenberg, Fundations of Modern Probability. Second edition. Springer-Verlag, New York (2001).

[14] D.E. Knuth, The art of computer programing, Volume 1: Fundamental algorithms. Second edition. Addison-Wesley, Reading, MA (1997).

[15] M. Kuba and A. Panholzer, The left-right-imbalance of binary search trees. Available at: http://info.tuwien.ac.at/ panholzer (2006).

[16] G. Louchard, Exact and asymptotic distributions in digital and binary search trees. RAIRO Theoret. Inform. Appl. 21 (1987) 479-496.

[17] H. Mahmoud, Evolution of Random Search Trees. John Wiley, New York (1992).

[18] H.M. Mahmoud and R. Neininger, Distribution of distances in random binary search trees. Ann. Appl. Prob. 13 (2003) $253-276$.

[19] H.M. Mahmoud and R.T. Smythe, A survey of recursive trees. Theoret. Probab. Math. Statist. 51 (1995) 1-27.

[20] J.-F. Marckert, The rotation correspondence is asymptotically a dilatation. Random Struct. Algorithms 24 (2004) 118-132.

[21] G. Slade and T. Hara, The scaling limit of the incipient infinite cluster in high-dimensional percolation. II. Integrated superbrownian excursion. J. Math. Phys. 41 (2000) 1244-1293. 